\title{
Método de la deformación unitaria para diseño de vigas rectangulares doblemente reforzadas
}

\author{
Strain method for doubly reinforced rectangular beams design \\ Método da deformação unitária para desenho de vigas \\ retangulares duplamente reforçadas
}

Fecha de Recepción: 03 de noviembre de 2014

Luis Rafael Prieto-Serrano*

Fecha de Aceptación: 12 de julio de 2015

\section{Resumen}

El acero en compresión en una viga doblemente reforzada mejora la ductilidad, reduce las deflexiones instantáneas y de largo plazo, está disponible para tomar la tracción en secciones sometidas a reversión de esfuerzos y, en vigas de poca altura, aumenta la resistencia. A partir de la deformación unitaria esperada del acero en tracción, como medida de la ductilidad de la sección, se propone una metodología directa para determinar las cantidades de refuerzo necesarias, tanto en compresión como en tracción. Se establece que las secciones más eficientes son aquellas para las cuales la deformación en el acero en tracción es 0.005 .

Palabras clave: Concreto estructural, Diseño de vigas, Deformación unitaria, Ductilidad, Viga doblemente reforzada.

\begin{abstract}
The compressed steel in a doubly reinforced beam improves ductility, reduces immediate and long-term deflections, it is available to support the tension in sections under reversal stress, and in low-height beams, it increases strength. With the expected strain as a measure of the section ductility, the paper proposes a direct methodology to determine the necessary compression quantities and tension reinforcement. It finds that the most efficient sections are those for which the tension reinforcing strain is 0.005 .
\end{abstract}

Key Words: Beams design, Doubly Reinforced Beam, Ductility, Strain, Structural Concrete.

* M.Sc. Universidad Pedagógica y Tecnológica de Colombia (Tunja-Boyacá, Colombia). luis.prieto@uptc.edu.co. 


\section{Resumo}

O aço em compressão em uma viga duplamente reforçada melhora a ductilidade, reduz as deflexões instantâneas e de longo prazo, está disponível para tomar a tração em seções submetidas a reversão de esforços e, em vigas de pouca altura, aumenta a resistência. A partir da deformação unitária esperada do aço em tração, como medida da ductilidade da seção, propõe-se uma metodologia direta para determinar as quantidades de reforço necessárias, tanto em compressão como em tração. Estabelece-se que as seções mais eficientes são aquelas para as quais a deformação no aço em tração é de 0.005 .

Palavras chave: Concreto estrutural, Desenho de vigas, Deformação unitária, Ductilidade, Viga duplamente reforçada. 


\section{INTRODUCCIÓN}

En la primera edición de este siglo del Reglamento $A C I$ 318 [1] se introdujeron modificaciones importantes en los factores de carga de las combinaciones de carga y en los factores de reducción de resistencia $\varphi$ utilizados en diseño; estos cambios fueron adoptados integralmente por el NSR-10 [2], que reemplazó las NSR-98 [3] y reformó la práctica de diseño de vigas en Colombia.

El factor de reducción de resistencia $\varphi$ contemplaba variaciones en la resistencia de los materiales y en las dimensiones de las secciones, defectos en la colocación del acero de refuerzo y aproximaciones en las hipótesis de diseño, y dependía de la importancia de la fuerza interna en cuanto a la consecuencia de la falla de los elementos "respecto a toda la estructura y el grado de advertencia implícita en el modo de falla" [4]. La práctica actual, además, contempla el grado de ductilidad de la sección, por lo que ahora $\varphi$ se determina "por las condiciones de deformación unitaria en las secciones transversales" [5].

Los cambios en la conceptualización del factor de reducción de resistencia permiten apartarse del control de cuantías del acero, necesarios en la práctica anterior, y concentrarse en la deformación unitaria del acero de refuerzo a tracción, como una medida más directamente relacionada con la ductilidad de las secciones de concreto reforzado.
Con un procedimiento como el que aquí se propone para vigas rectangulares de concreto estructural doblemente reforzadas se determinan directamente las cantidades de acero necesarias en compresión y en tracción.

\section{El PROBlemA de diseño}

El diseño estructural tiene como uno de sus objetivos proveer elementos dúctiles, con el fin de desarrollar una buena capacidad de disipación de energía; hoy en día, para cumplir con este propósito se debe garantizar que cuando se alcance la resistencia de la viga en la sección crítica, el acero longitudinal extremo en tracción debe presentar mínimo una deformación unitaria $\varepsilon_{\mathrm{t}}$ de $0.004[2,5]$.

Para una sección rectangular de concreto doblemente reforzada por $\mathrm{A}_{\mathrm{s}} \mathrm{y} \mathrm{A}_{\mathrm{s}}^{\prime}$ (Fig. 1.a) sometida a la acción de un momento flector mayorado de diseño $M_{u}$ (Fig. 1.b) se define la altura efectiva d como "la distancia desde la fibra extrema en compresión hasta el centroide del refuerzo longitudinal en tracción" [2], $\mathrm{y}$, similarmente, d' como "la distancia desde la fibra extrema en compresión hasta el centroide del refuerzo longitudinal en compresión" [2]. En la práctica de diseño de vigas de concreto estructural estas alturas se aproximan al centímetro $[2,5]$.

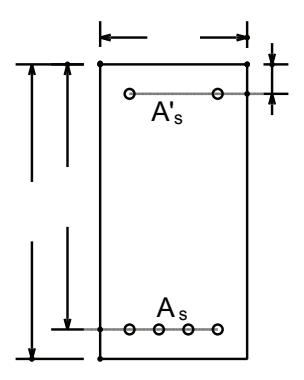

(a)

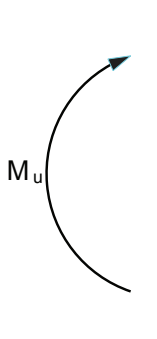

FIG. 1. Viga doblemente reforzada en el estado limite de resistencia.
(a) Sección transversal (b) Sección longitudinal (c) Diagrama de deformaciones (d) Diagrama de esfuerzos (e)

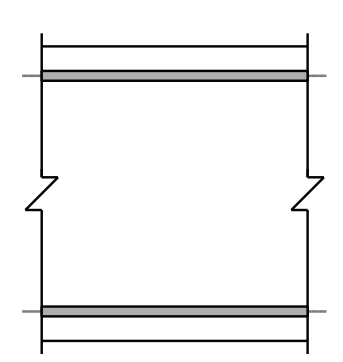

(b)

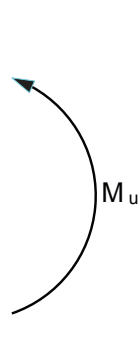

(c)

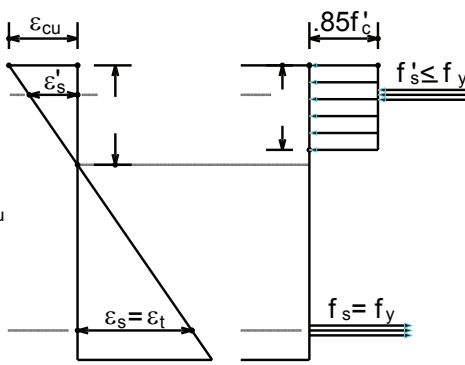

(d)

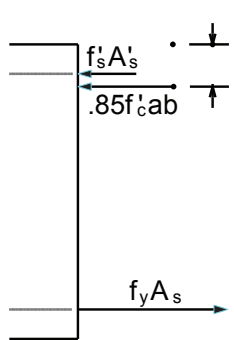

(e) Diagrama de fuerzas.

Teniendo presentes las suposiciones de diseño de elementos de concreto reforzado, ampliamente documentadas $[4,6,7]$ :
- Se parte de la hipótesis de deformación de Bernoulli-Euler, mediante la cual secciones planas antes de la flexión permanecen planas después de ella (Fig. 1.c). Esto permite establecer 
c, la frontera entre las fibras en tracción y las de compresión.

- No se considera la zona de endurecimiento por deformación del acero de refuerzo, al definir su comportamiento, en tracción y en compresión, con una curva $f-\varepsilon$ perfectamente elastoplástica (Fig. 2).

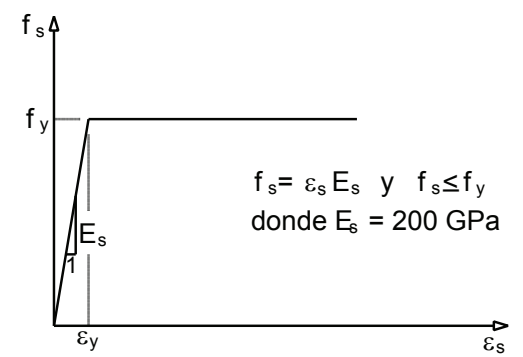

Fig. 2. Curva f- $\varepsilon$ del acero de refuerzo.

- Se desprecia la contribución del concreto para resistir tracciones.

- Para evaluar la resistencia del concreto en compresión se utiliza el método de Whitney [8] en reemplazo de la "forma general de la curva $f-\varepsilon$ del concreto" [5] (ver Fig. 3 con ejes intercambiados), que facilita el diseño sin modificar la sensibilidad de la magnitud de la resistencia. $\beta_{1}$ es un factor de forma "que relaciona la profundidad del bloque rectangular equivalente de esfuerzos con la profundidad del eje neutro" [2].

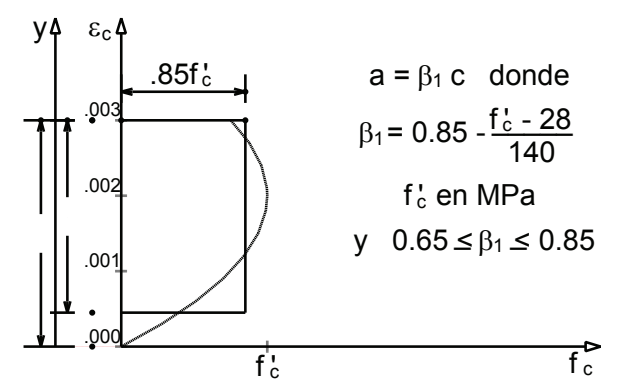

Fig. 3. Curva f- $\varepsilon$ equivalente del concreto, método de Whitney.
- Mediante pruebas de laboratorio se ha demostrado que la resistencia de la sección se alcanza cuando falla el concreto, haya fluido o no el acero a tracción $[4,6,7]$. Para efectos de diseño se ha establecido que la falla del concreto se presenta cuando la fibra extrema alcanza una deformación $\varepsilon_{\text {cu }}$ de 0.003 [2], y el acero a tracción fluye.

Al momento de alcanzarse la resistencia, el diagrama de esfuerzos en la sección crítica de la viga corresponderá al que se observa en la Fig. 1.d.

Integrando cada uno de estos esfuerzos en las respectivas áreas en que actúan, las fuerzas obtenidas se muestran en la Fig. 1.e. La resultante de estas fuerzas será un par $\mathrm{M}_{\mathrm{n}}$, momento nominal resistente, que en diseño debe ser tal que:

$$
\mathrm{M}_{\mathrm{u}} \leq \phi \mathrm{M}_{\mathrm{n}}(1)
$$

donde $\phi$ es el factor de reducción de resistencia, que toma en cuenta variaciones en las calidades de los materiales y en la mano de obra, en cuanto dimensiones de la sección y colocación del acero de refuerzo y el tipo de falla que se puede llegar a presentar en el elemento y su importancia en la estructura [5]. En la práctica actual de diseño $\phi$ depende de la deformación unitaria del acero de refuerzo extremo en tracción \&t $[2,5]$.

\section{RESISTENCIA NOMINAL RESISTENTE DE SECCIONES DÚCTILES DOBLEMENTE REFORZADAS}

Para que una sección de concreto reforzado sometida a flexión sea dúctil es necesario que el acero de refuerzo a tracción se deforme de tal manera que esté trabajando en el rango plástico al alcanzar el estado límite de resistencia. Así, la deformación unitaria del acero a tracción debe ser $\varepsilon_{\mathrm{s}}>\varepsilon_{\mathrm{y}}$, por lo que la sección, al alcanzar la resistencia, se garantiza que el esfuerzo en el acero a tracción es el límite de fluencia $\mathrm{f}_{\mathrm{y}}$ (Fig. 1.d). De otro lado, el acero a compresión puede haber fluido o no. 


\section{A. Caso I. En el límite de resistencia, el acero a compresión fluye}

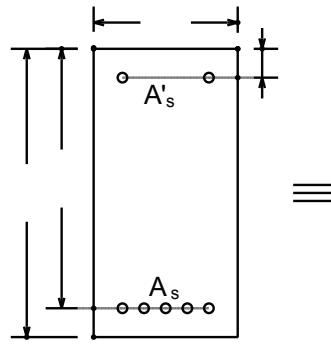

(a)

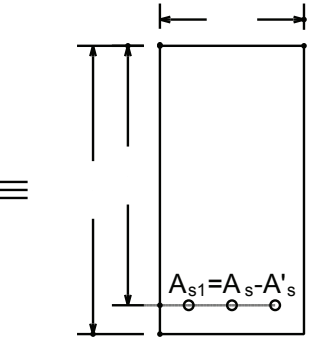

(b)

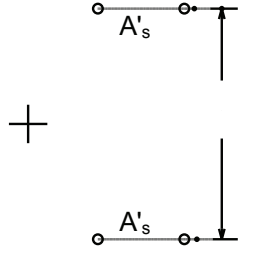

(c)

Fig. 4. Sección equivalente de una viga doblemente reforzada para el caso que el acero a compresión fluya en el estado límite de resistencia.

Si en el estado límite de resistencia tanto el acero a tracción como el de compresión fluyen, al determinar el momento nominal resistente $M_{n}$ es usual considerar la sección doblemente reforzada $\mathrm{A}_{\mathrm{s1}}=\mathrm{A}_{\mathrm{s}}$-A's (Fig. 4.a) como el equivalente a la sección reforzada con una cantidad de acero a tracción igual a (Fig. 4.b) más la acción de la cantidad de acero en compresión A's actuando tanto en las fibras superiores como inferiores (Fig. 4.c). Así, la resistencia nominal de la sección se puede evaluar como:

$$
\mathrm{M}_{\mathrm{n}}=\mathrm{M}_{\mathrm{n} 1}+\mathrm{M}_{\mathrm{n}}^{\prime}
$$

en el que $\mathrm{M}_{\mathrm{n} 1}$ es el momento nominal resistente de la sección simplemente reforzada con $\mathrm{A}_{\mathrm{S} 1}$ :

$$
\mathrm{M}_{\mathrm{n} 1}=\phi \rho_{1} \mathrm{f}_{\mathrm{y}}\left(1-\frac{\mathrm{m}}{2} \rho_{1}\right) \mathrm{b} \mathrm{d}^{2}
$$

donde $\rho_{1}$ es la cuantía de la sección simplemente reforzada $\left(\mathrm{A}_{\mathrm{s1}}\right.$ bd $)$ y $\mathrm{m}$ la relación de resistencias de los materiales constitutivos de la viga: $\mathrm{f}_{\mathrm{y}} / 0.85 \mathrm{f}_{\mathrm{c}}$.

Similarmente, $\mathrm{M}_{\mathrm{n}}^{\prime}$ corresponderá al momento del par de fuerzas que se presentan en el refuerzo $\mathrm{A}_{\mathrm{s}}^{\prime}$ tanto en las fibras superiores como inferiores:

$$
\mathrm{M}_{\mathrm{n}}^{\prime}=\mathrm{A}_{\mathrm{s}}^{\prime} \mathrm{f}_{\mathrm{y}}\left(\mathrm{d}-\mathrm{d}^{\prime}\right)
$$

Se demuestra que el acero en compresión fluye cuando la cuantía $\rho_{1}$ es

$$
\rho_{1} \geq \frac{\beta_{1}}{\mathrm{~m}}\left(\frac{600}{600-\mathrm{f}_{\mathrm{y}}}\right) \frac{\mathrm{d}^{\prime}}{\mathrm{d}}(5)
$$

Si la desigualdad (5) no se cumple, el acero en compresión no fluye, lo que se presenta en el apartado B.

\section{B. Caso II. En el límite de resistencia, el acero a compresión no fluye.}

En secciones dúctiles doblemente reforzadas aquellas que en la resistencia el acero a tracción alcanza la fluencia- para las que el acero a compresión no fluye, es decir, pueden establecer dos ecuaciones del esfuerzo del acero en compresión en función de la profundidad del bloque rectangular equivalente de esfuerzos del concreto. Una de ellas a partir del diagrama de deformaciones (Fig. 1.c),

$$
\mathrm{f}_{\mathrm{s}}=600\left(\frac{\mathrm{a}-\beta_{1} \mathrm{~d}^{\prime}}{\mathrm{a}}\right)
$$

y la otra del equilibrio de fuerzas axiales (Fig. 1.e):

$$
\mathrm{A}_{\mathrm{s}} \mathrm{f}_{\mathrm{y}}=0.85 \mathrm{f}_{\mathrm{c}}^{\mathrm{a}} \mathrm{ab}+\mathrm{A}_{\mathrm{s}}^{\prime} \mathrm{f}_{\mathrm{s}}
$$

Reemplazando la ecuación (6) en (7), da como resultado un polinomio de segundo grado en función de a, que sería la única incógnita. Así, el momento nominal resistente será:

$$
\mathrm{M}_{\mathrm{n}}=0.85 \mathrm{f}^{\prime}{ }_{\mathrm{c}} \mathrm{ab}(\mathrm{d}-) \frac{\mathrm{a}}{2}+\mathrm{A}_{\mathrm{s}}^{\prime} \mathrm{f}_{\mathrm{s}}\left(\mathrm{d}-\mathrm{d}^{\prime}\right)
$$

\section{EXPRESIONES PARA DISEÑO}

La deformación del concreto $\varepsilon_{\text {cu }}$ ha sido fijada en el diagrama de deformaciones (Fig. 1.c) para el estado límite de la resistencia en 0.003 . Se observa que no solo 
la posición del ejeneutroc, sino también la deformación unitaria al centroide del acero en compresión $\varepsilon_{\text {s', }}$, dependen del valor de $\varepsilon_{\mathrm{s}}$ (la deformación unitaria en el centroide del acero longitudinal del refuerzo en tracción) o de $\varepsilon_{t}$, que para este caso es igual. Conocida la deformación, se determina el esfuerzo por lo que en la condición crítica de diseño $\mathrm{M}_{\mathrm{u}}=\phi \mathrm{M}_{\mathrm{n}}$, del par resultante de las fuerzas que actúan en la sección (Fig. 1.e), se deduce que:

$$
\mathrm{A}_{\mathrm{s}}^{\prime} \geq \frac{\frac{\mathrm{M}_{\mathrm{u}}}{\phi}-0.85 \mathrm{f}_{\mathrm{c}} \mathrm{ab}\left(\mathrm{d}-\frac{\mathrm{a}}{2}\right)}{\mathrm{f}_{\mathrm{s}}^{\prime}\left(\mathrm{d}-\mathrm{d}^{\prime}\right)}
$$

y del equilibrio de fuerzas

$$
\mathrm{A}_{\mathrm{s}} \geq \frac{\mathrm{ab}}{\mathrm{m}}+\mathrm{A}_{\mathrm{s}}^{\prime} \frac{\mathrm{f}_{\mathrm{s}}^{\prime}}{\mathrm{f}_{\mathrm{y}}}(10)
$$

\section{EL DISEÑo}

Conociendo las dimensiones de la viga, en general resultantes de un análisis de rigidez de la estructura por diseñar, se especifica la resistencia del concreto $f^{\prime}{ }_{c} y$ el límite de fluencia del acero de refuerzo longitudinal $\mathrm{f}_{\mathrm{y}}$, para que resista el momento mayorado crítico $\mathrm{M}_{\mathrm{u}}$ resultante de las combinaciones de carga que puedan actuar durante su vida útil.

Como usualmente se hace, el diseño de secciones de concreto reforzado parte de suponer que la viga es simplemente reforzada, si la solución presenta una cuantía mayor que la permitida -y si no es conveniente o posible cambiar de dimensiones o si es poco práctico modificar especificaciones de materiales- existirá siempre la posibilidad de colocar acero en compresión.

El diseño de secciones rectangulares doblemente reforzadas -a partir del recubrimiento necesario y de estimar los diámetros del acero transversal y longitudinal- inicia por suponer la altura efectiva de la viga $\mathrm{d}$ y la posición del acero de refuerzo en compresión d'; de fijar un criterio de deformación unitaria del acero longitudinal extremo en tracción $\varepsilon_{t} \mathrm{y}$ de que la cantidad de acero necesaria para reforzar la viga, tanto en tracción como en compresión, se pueda emplazar cada una en una fila de refuerzo.

Si esto último es factible, es la solución más económica. En este caso la posición del centroide del acero en tracción d será igual a $\mathrm{d}_{\mathrm{t}}$, que es la "distancia desde la fibra extrema en compresión al centroide de la fila extrema de acero longitudinal en tracción" $[2,5]$. De esta manera, $\varepsilon_{\mathrm{s}}=\varepsilon_{\mathrm{t}}$, lo que permite determinar todos los valores de las expresiones para diseño del punto anterior de una manera directa, incluidas las áreas de refuerzo necesarias en compresión y en tracción.

$\mathrm{Si}$, por el contrario, el área de acero necesaria en tracción solo es posible colocarla en dos o más filas $\mathrm{d}<\mathrm{d}_{\mathrm{t}}$ y $\varepsilon_{\mathrm{s}}<\varepsilon_{\mathrm{t}}$ (Fig.5), con base en la Fig.5.c es posible determinar la posición del eje neutro para esta condición.

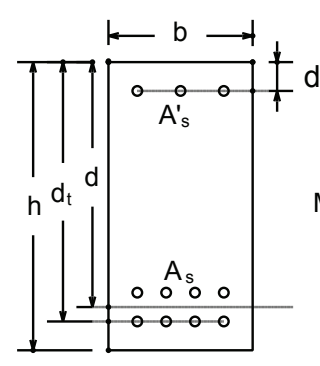

(a)

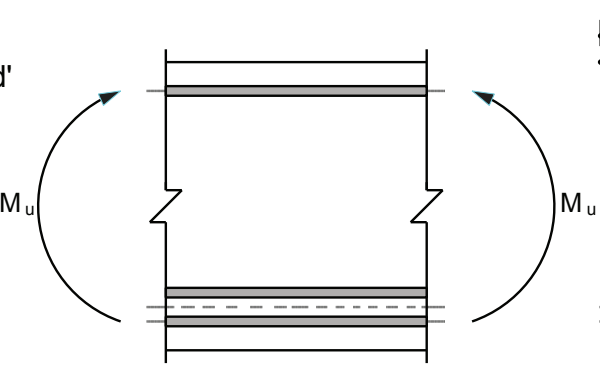

(b)

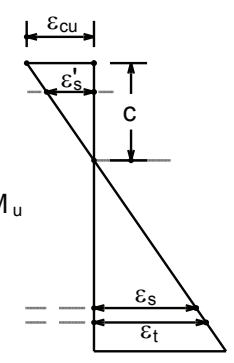

(c)

Fig. 5. Viga de concreto doblemente reforzada mediante dos capas de acero de refuerzo a tracción.

(a) Sección transversal (b) Sección longitudinal (c) Diagrama de deformaciones.

Como las áreas de refuerzo en diseño no se pueden colocar 'exactamente' al definir el número y diámetro de las barras a usar, se recomienda elegirlas ligeramente mayores que los valores determinados para $\mathrm{A}_{\mathrm{s}} \mathrm{y} \mathrm{A}_{\mathrm{s}}^{\prime}$, teniendo especial cuidado para que $\Delta \mathrm{A}_{\mathrm{s}}^{\prime}>\Delta \mathrm{A}_{\mathrm{s}}$, con el 
fin de que $\varepsilon_{\mathrm{t}}$ sea mayor o similar al valor previsto de diseño.

Las expresiones de diseño (9) y (10) no toman en cuenta el área de concreto desplazada por el refuerzo en compresión; aunque sería más 'exacto', considerarla no cambia significativamente el momento resistente de la sección. Esto último es más cierto en la medida en que $\varepsilon_{\mathrm{t}} \leq 0.005$, y el momento resistente esté cercano al que puede resistir la viga con el máximo refuerzo permitido para la sección simplemente reforzada, donde la cantidad de acero en compresión es relativamente pequeña. En caso de que se aleje de estas condiciones y se considere necesario tomar en cuenta el área de concreto desplazada por el acero en compresión, el área de acero en compresión aumentaría y la de tracción permanecería constante.

Para los casos (excepcionales) que $\mathrm{f}_{\mathrm{s}} \leq 0$ la altura de la sección será insuficiente para las condiciones de diseño $y$, en la medida que este esfuerzo sea pequeño ( $\varepsilon t \gg 0.005 \mathrm{y} \mathrm{M}_{\mathrm{u}}$ elevado), la sección tenderá a ser ineficiente, pues demandará grandes cantidades de acero.

\section{EFICIENCIA DE LA SECCIÓN}

El diseño debe definir la deformación unitaria que se espera que presente la fila extrema del acero de refuerzo en tracción de la viga en el estado límite de resistencia, la cual es una medida de la ductilidad de la sección e, indirectamente, de la capacidad de disipación de energía de la sección, "el límite de 0.005 proporciona un comportamiento dúctil para la mayoría de los diseños" [5].En casos especiales, como en aquellos sitios donde se permita la redistribución de momentose, $e_{t}$ como mínimo debe ser $0.0075[2,5]$.
Para el caso de diseño de una viga de dimensiones constantes $(0.30 \mathrm{x} 0.45 \mathrm{~m})$ y materiales conocidos $\left(\mathrm{f}_{\mathrm{c}}{ }_{\mathrm{c}}=21 \mathrm{MPa}\right.$ y $\mathrm{f}_{\mathrm{y}}=420 \mathrm{MPa}$ ), el área de refuerzo total -la de tracción más la de compresión- necesaria para resistir un momento mayorado dado varía sensiblemente con la deformación unitaria requerida del acero longitudinal extremo de tracción $\varepsilon_{\mathrm{t}}$. En la Fig. 6 se puede observar el área total teórica requerida para algunos momentos mayorados de diseño en función de $\varepsilon_{\mathrm{t}}$. En todos los casos, la menor cantidad de acero total que necesita la sección se presenta cuando $\varepsilon_{\mathrm{t}}=0.005$.

Para valores entre $0.004 \leq \varepsilon_{\mathrm{t}}<0.005$, las cantidades de acero totales son mayores que para deformaciones unitarias de 0.005 , debido al factor de reducción de resistencia $\varphi$ que modifica al momento nominal resistente y que, a partir del ACI 318-02 [7], es función de $\varepsilon_{\mathrm{t}}$ y lo disminuye tan sensiblemente, por lo que en algunos casos demanda áreas totales superiores al $10 \%$ para deformaciones al límite de 0.004 . Por esta razón, no existe justificación alguna-de comportamiento o económica- para diseñar secciones en este rango de deformaciones unitarias para el acero a tracción.

Por otro lado, si las deformaciones unitarias en el acero longitudinal extremo $\varepsilon_{\mathrm{t}}>0.005$, las áreas totales necesarias pueden ser mucho mayor es. Los casos mostrados pueden bordear el $30 \%$, con $\varepsilon_{\mathrm{t}}=0.0075$, si $\mathrm{M}_{\mathrm{u}}$ es cercano al que puede resistir la viga con el máximo refuerzo permitido para la sección simplemente reforzada.En la medida que los momentos sean mayores, estas proporciones de acero total van disminuyendo, aunque, como se mencionó, para todos los casos las secciones más económicas son aquellas para las cuales $\varepsilon_{\mathrm{t}}=0.005$, pues la necesidad de proveer mayor ductilidad de la sección demandará mayores cantidades de acero tanto en compresión como en tracción. 


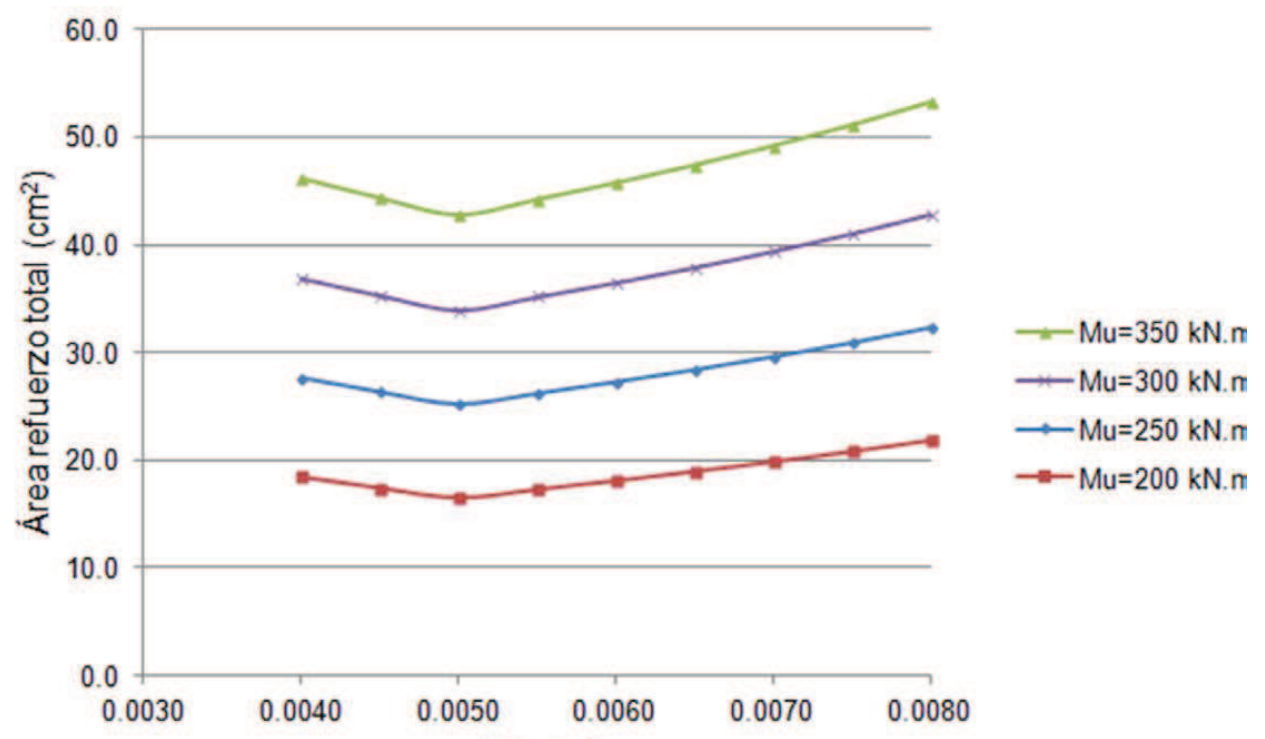

Fig. 6. Caso viga $0.30 \times 0.45, \mathrm{f}^{\prime}{ }_{\mathrm{c}}=21 \mathrm{MPa} \mathrm{y} \mathrm{f}_{\mathrm{y}}=420 \mathrm{MPa}$.

\section{EJEMPLO}

Una viga rectangular apoyada, simplemente, de 5.50 $\mathrm{m}$ de luz debe soportar una carga viva de servicio de $36.0 \mathrm{kN} / \mathrm{m}$, además de una carga permanente de $15.3 \mathrm{kN} / \mathrm{m}$. Por razones arquitectónicas, la sección transversal está limitada a un ancho de $0.25 \mathrm{~m}$ y a una altura de $0.50 \mathrm{~m}$.Si el acero de refuerzo por usar tiene un límite de fluencia de $420 \mathrm{MPa}$, y el concreto, una resistencia especificada de $28 \mathrm{MPa}$, ¿cuál es el área (o áreas) de acero que debe(n) suministrarse?

La carga mayor a da de diseño es $1.2 * 15.3+1.6 * 36.0=76.0 \mathrm{kN} / \mathrm{m}$.

Así, $\mathrm{Mu}=76.0^{*} 5.50^{2} / 8=287 \mathrm{kN} \cdot \mathrm{m}$. Para garantizar los requisitos de recubrimiento y espaciamiento del refuerzo a tracción se supone que su centroide está a $90 \mathrm{~mm}$ por encima de la cara inferior de la viga; de esta forma, $\mathrm{d}=0.41 \mathrm{~m}$; se prevé que el centroide del acero de refuerzo extremo está a $70 \mathrm{~mm}$ de la cara inferior $\mathrm{y} \mathrm{d}_{\mathrm{t}}=0.43 \mathrm{~m}$. Para las calidades de acero y concreto de diseño, $m=17.6$. Si la viga fuese simplemente reforzada, la cuantía necesaria sería:

$\rho=\frac{1}{17.6}\left[1-\sqrt{1-\frac{2 * 17.6 * 287}{0.9 * 0.25 * 0.41^{2} * 420 * 10^{3}}}\right]=0.0225$

mayor que $0.0181 * 0.43 / 0.41=0.0190$-cuantía que corresponde a una $\varepsilon_{\mathrm{t}}=0.005-$, lo que significa que es necesario reforzar la viga a compresión. Para este caso, con una deformación unitaria del acero extremo en tracción de 0.005 , la altura de esfuerzos constantes en el concreto será:

$$
\mathrm{a}=\frac{0.003}{0.003+0.005} 0.85 * 430=137 \mathrm{~mm}
$$

Si el centroide del acero a compresión está ubicado a $60 \mathrm{~mm}$ por debajo de la cara superior de la viga $(\mathrm{m})$, el esfuerzo al que estará sometido este acero valdrá (6):

$$
\mathrm{f}_{\mathrm{s}}^{\prime}=600\left(1-\frac{0.85 * 60}{137}\right)=377 \mathrm{MPa}<420 \mathrm{MPa}
$$

De la ecuación (9),

$$
\mathrm{A}_{\mathrm{S}}^{\prime}=\frac{\frac{287^{*} 10^{6}}{0.90}-0.85 * 28 * 137 * 250 *\left(410-\frac{137}{2}\right)}{377(410-60)}=310 \mathrm{~m} \mathrm{~m}^{2}
$$


$\mathrm{y}$ de (10),

$$
\mathrm{A}_{\mathrm{s}}=\frac{137 * 250}{17.6}+310 * \frac{377}{420} 2220 \mathrm{~m} \mathrm{~m}^{2}
$$

Se usan $3 \mathrm{~N}^{\circ} 8$ y $2 \mathrm{~N}^{\circ} 7$ con un área de $2300 \mathrm{~mm}^{2}$ para el acero de refuerzo a tracción dispuestos de la forma como se observa en la Fig.7, se verifica d y se determina $\rho=0.0224$. Como refuerzo a compresión se emplean $2 \mathrm{~N}^{\circ} 5$ con un área de $400 \mathrm{~mm}^{2}$ y un $\rho^{\prime}=0.0039$.

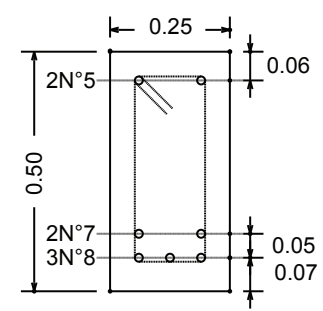

Fig. 7. Solución de refuerzo para la viga del ejemplo.
Se verifica que el refuerzo colocado a la viga es suficiente para resistir el momento de diseño. $\rho-\rho^{\prime}=0.0185$. Como es menor que (5):

$$
\frac{0.85}{17.6}\left(\frac{600}{600-420}\right) \frac{60}{410}=0.0236
$$

el acero a compresión no fluye al momento que la viga alcance la resistencia. Para este caso, al determinar 'exactamente' la altura del bloque de esfuerzos en el concreto al resolver el polinomio de segundo grado,

$\frac{0.85 * 28 * 250}{400} \mathrm{a}^{2}+\left(600-\frac{2300}{400} * 420\right) \mathrm{a}-600 * 0.85 * 60=0$
$\mathrm{a}=137 \mathrm{~mm}$ y con este, como se determinó
anteriormente, $\mathrm{f}_{\mathrm{s}}^{\prime}=377 \mathrm{MPa}$. Se verifica así que

$$
\varepsilon_{\mathrm{t}}=0.003 \frac{0.85 * 430-137}{137}=0.00500
$$

$$
\begin{gathered}
\text { Por lo que y de (8): } \\
\phi \mathrm{M}_{\mathrm{n}}=0.90\left[0.85 * 28 * 137 * 250\left(410-\frac{137}{2}\right)+400 * 377(410-60)\right\} 10^{6}=298 \mathrm{kN} \cdot \mathrm{m}
\end{gathered}
$$

algo mayor que $287 \mathrm{kN} \cdot \mathrm{m}$.

\section{Conclusiones}

El método de la deformación unitaria propuesto, para el diseño de vigas rectangulares de concreto estructural doblemente reforzadas, permite determinar las cantidades de acero de refuerzo en compresión y en tracción mediante un procedimiento directo.

Se determinó que en el diseño de vigas reforzadas a compresión, la deformación unitaria óptima que presente el acero de refuerzo extrema en tracción en el estado límite de resistencia es de 0.005. En casos especiales, como en aquellos sitios donde se permita la redistribución de momentos, para los cuales sea necesario garantizar una e mínimo de 0.0075 , la demanda de acero aumenta sensiblemente, cercana al 30\% mayor respecto de la necesaria para una deformación unitaria de 0.005 .

Considerar secciones en el límite de resistencia con deformaciones unitarias menores a 0.005 es inconveniente debido a la reducción que se presenta en la ductilidad de la sección. La normatividad existente toma en consideración este comportamiento,disminuyendo el factor de reducción de resistencia $\varphi$, lo que se refleja en mayores demandas de refuerzo, afectando su viabilidad económica.

\section{REFERENCIAS}

[1] Committee ACI 318-American Concrete Institute, Building Code Requirements for Structural Concrete (ACI 318-02) and Commentary (ACI 318R-02), Farmington Hills, Michigan, American Concrete Institute, 2002, pp. 85-118.

[2] Asociación Colombiana de Ingeniería Sísmica, "Título C - Concreto estructural". En Reglamento Colombiano de Construcción Sismo Resistente - NSR-10, Bogotá, Colombia, Asociación Colombiana de Ingeniería Sísmica, 2010, pp. C1-C227.

[3] Asociación Colombiana de Ingeniería Sísmica, "Título C - Concreto estructural". En Normas Colombianas de Diseño y Construcción Sismo Resistente - NSR-98, Bogotá, Colombia, Asociación Colombiana de Ingeniería Sísmica,1998, pp. C1-C588. 
[4] R. Park, T. Paulay, Estructuras de concreto reforzado, Tr. S. Fernández, México D.F., México: Limusa S. A., 1983.

[5] Comité ACI 318-American Concrete Institute, Requisitos de Reglamento para Concreto Estructural (ACI 318S-11) y Comentario, Tr. Subcomité 318-S - American Concrete Institute, Farmington Hills, Michigan, American Concrete Institute, 2011.
[6] A. Nilson, D. Darwin, C. Dolan, Design of concrete structures, McGraw-Hill, 2010.

[7] O. González, O. Robles, Aspectos fundamentales del concreto reforzado, México D.F., México:Limusa S. A., 2005.

[8] C. S. Whitney, "Design of reinforced concrete members under flexure or combined flexure and direct compression", J. ACI, vol. 33, pp. 483498, Mar.-Apr. 1937. 